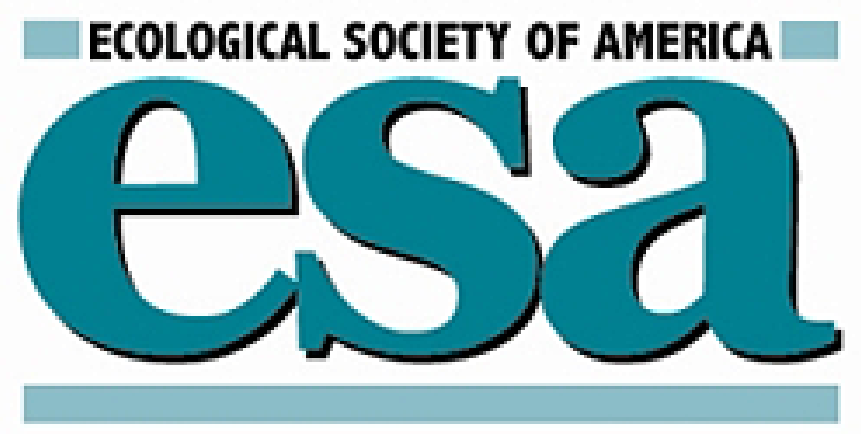

Body Size, Energy Use, and Community Structure of Small Mammals Author(s): S. K. Morgan Ernest

Source: Ecology, Vol. 86, No. 6 (Jun., 2005), pp. 1407-1413

Published by: Ecological Society of America

Stable URL: http://www.jstor.org/stable/3450766

Accessed: 02/04/2014 15:47

Your use of the JSTOR archive indicates your acceptance of the Terms \& Conditions of Use, available at http://www.jstor.org/page/info/about/policies/terms.jsp

JSTOR is a not-for-profit service that helps scholars, researchers, and students discover, use, and build upon a wide range of content in a trusted digital archive. We use information technology and tools to increase productivity and facilitate new forms of scholarship. For more information about JSTOR, please contact support@jstor.org. 


\title{
BODY SIZE, ENERGY USE, AND COMMUNITY STRUCTURE OF SMALL MAMMALS
}

\author{
S. K. Morgan ERnest ${ }^{1}$ \\ Department of Biology, University of New Mexico, Albuquerque, New Mexico 87131 USA
}

\begin{abstract}
Body size has long been hypothesized to play a major role in community structure and dynamics. Two general hypotheses exist for how resources are distributed among body sizes: (1) resources are equally available and uniformly utilized across body sizes and (2) resources are differentially available to organisms of different body sizes, resulting in a nonuniform or modal distribution. It has also been predicted that the distribution of body sizes of species in a community should reflect the underlying availability of resources, with the emergence of aggregations of species around specific body sizes. I examined the relationship between energy utilization, body size, and community structure in nine small-mammal communities in North America. In all communities, energy use across body sizes was significantly different from uniform. In contrast, none of the nine species-level body size distributions were significantly different from uniform. Cross-site comparisons showed that, while the species-level body size distribution did not vary significantly among sites, the utilization of energy across body sizes did. These results suggest that uniform energy utilization does not occur in small-mammal communities and that the species-level body size distribution of a community is not determined by resource utilization.
\end{abstract}

Key words: body size distribution; body size-energy distribution; community structure; energetic equivalence rule; macroecology; small mammals.

\section{INTRODUCTION}

Understanding the role of organismal size in the structure of communities has been a major research focus in community ecology since Hutchinson (1959) introduced the idea of body size ratios (i.e., that potentially competing species should differ in body size, such as a difference in length by a factor of about 1.3 or more). While subsequent studies have found little support for the idea that body size ratios reflect strong processes structuring communities (Istock 1973, Horn and May 1977, Strong et al. 1979), the importance of addressing whether the size of organisms affects the assembly of communities or the partitioning of resources has not diminished. One approach used to address these questions has been to examine the frequency distribution of body sizes of species in a community (Hutchinson and MacArthur 1959). These distributions, referred to here as species-level body size distributions (BSD), provide insights into community assembly and the ability of species of similar size to coexist in communities (e.g., Hutchinson and MacArthur 1959, Brown and Nicoletto 1991, Gaston and Blackburn 2000). However, because this pattern does not consider species' abundances, it provides only a

Manuscript received 8 December 2003; revised 16 November 2004; accepted 4 December 2004. Corresponding Editor: F. S. Dobson.

${ }^{1}$ Present address: Department of Biology, Utah State University, Logan, Utah 84322 USA.

E-mail: morgane@biology.usu.edu highly simplified view of resource partitioning. A second approach to understanding the importance of size has been to assess, both within and across communities, the relationship between the average mass of a species and its abundance (e.g., Damuth 1981, Blackburn et al. 1993, Russo et al. 2003). Because this relationship often suggests that energy use is equivalent across body sizes (by combining the abundance of a species with its metabolic rate) it is referred to as the energetic equivalence rule (EER; e.g., Damuth 1981, Marquet et al. 1990, Ernest et al. 2003). However, this pattern has had mixed support within communities (e.g., Blackburn et al. 1993, Blackburn and Lawton 1994, Silva and Downing 1995, Russo et al. 2003) and does not take into account the relationship between body size and species richness. It is therefore unclear what insights the EER provides into the role of body size in the partitioning of resources within communities (Gaston and Blackburn 2000). A third approach typically assesses the role of body size in resource partitioning by determining the proportion of total community abundance or biomass occurring within different body size classes, without regard to species identity. This approach has been referred to as the size spectrum, and while it has been used extensively in aquatic systems (e.g., Sheldon et al. 1972, Griffiths 1986, Cyr and Pace 1993, Kerr and Dickie 2001), these analyses are only rarely conducted in terrestrial systems (but see Morse et al. 1988, Stork and Blackburn 1993, White et al. 2004). 
In studies of terrestrial plants and animals, there are two predominant hypotheses for how resources are partitioned among body sizes. The first hypothesis, based on the EER, is that energy will be divided relatively equally among body sizes. Though traditionally the EER is a species-based pattern, some studies (using methodology similar to the size spectra approach) have shown that the total energy use by a body size can be invariant across body sizes (e.g., Enquist and Niklas 2001, Ackerman et al. 2004). The other hypothesis for how energy is partitioned with respect to size suggests that the distribution of energy use should be modal or multi-modal (Holling 1992), where a multimodal distribution is defined as a distribution containing more than one local maximum or peak (sensu Sokal and Rohlf 1995). While multimodal abundance and biomass distributions have been reported (Sheldon et al. 1972, Griffiths 1986, Cyr and Pace 1993), abundance and biomass do not necessarily directly reflect energy use within a community (e.g., Pagel et al. 1991, Taper and Marquet 1996, Thibault et al. 2004, White et al. 2004), and the prevalence of multimodal energy distributions in terrestrial animal communities is unclear.

Using the idea that energy is utilized unequally by different body sizes, it has been proposed that multimodal energy use should have a strong structuring effect on the species-level body size distribution of a community. If energy is unequally available across body sizes, then the body sizes that should be favored in the assembly of the community are those body sizes with the greatest access to resources, resulting in aggregations of species in the species-level body size distribution around specific masses (Holling 1992). While studies examine and debate the predicted aggregations in BSDs (Manly 1996, Lambert and Holling 1998, Allen et al. 1999, Siemann and Brown 1999, Forys and Allen 2002), there has been no examination of the relationship between the species-level body size distribution and the distribution of energy among body size classes (referred to henceforth as the body sizeenergy distribution or BSED).

Here, I use small-mammal communities to assess the two proposed patterns for energy use through communities. Specifically, I address the following questions: (1) Is energy use across body size categories uniform or multimodal? and (2) If energy use is not uniform across body sizes, does the species-level body size distribution of a community correspond to energy use throughout the community as postulated by Holling (1992)?

\section{METHods}

\section{Study areas and small-mammal data}

Data on small-mammal communities were obtained from several long-term studies in North America. For the purposes of this study, a small-mammal community is defined as all terrestrial mammals with an average body size $<400 \mathrm{~g}$ that were living within a predefined study area. In each data set, small mammals were sampled for more than one year and individuals were identified to species and weighed, yielding an extensive survey of the community. Multiple years of data were pooled (i.e., for the BSD, body sizes for each species were means taken across all sampled individuals and years, and for the BSED, every individual with a body mass that was captured during the time period of the study was used). Habitats ranged from alpine tundra to Chihuahuan desert shrubland, though a majority of the sites in this study come from the southwest United States. Only unmanipulated communities were used.

Andrews Long-Term Ecological Research (LTER) study area.-Located in the central Cascade Range of Oregon, this study area is a temperate coniferous forest habitat in the Willamette National Forest. Small mammals were censused (a combination of Sherman live traps and pitfall traps) in the fall season from 1995 to 1999 to assess small-mammal response to litter depth. This community consists of 9 species with body sizes ranging from an average of $4.46 \mathrm{~g}$ to $140 \mathrm{~g}$. More details on the study area and trapping methods are available online. ${ }^{2}$

Niwot LTER.-Located approximately $35 \mathrm{~km}$ west of Boulder, Colorado, this study area is an alpine tundra habitat in the Rocky Mountains. Small mammals were monitored (Sherman and Tomahawk live traps) at the study area during the summers from 1981 to 1990 . This community consists of 11 species with body sizes ranging from $4.0 \mathrm{~g}$ to $194 \mathrm{~g}$. More details on the study area and trapping methods are available online. ${ }^{3}$

Sevilleta LTER.-Located approximately $80 \mathrm{~km}$ south of Albuquerque, New Mexico, this 100000 -ha study area contains a variety of Chihuahuan Desert habitats. Within the Sevilleta, six distinct sites were censused: an arid grassland, two different Chihuahuan Desert creosote shrublands (separated by $>15 \mathrm{~km}$ and a river), a dune habitat with mixed shrubs and grasses, a juniper woodland, and a higher elevation piñon-juniper woodland. Small mammals were censused twice yearly at these sites from 1989 to 1998 using Sherman live traps. A total of 25 species were found on the Sevilleta with body sizes ranging from $7.37 \mathrm{~g}$ to 152 g. For details, see Ernest et al. (2000) and information available online. ${ }^{4}$

Portal project.-Located approximately $7 \mathrm{~km}$ from Portal, Arizona, this study area is an upper elevation Chihuahuan Desert habitat of mixed grasses and shrubs. Small mammals have been censused monthly since 1977 using Sherman live traps to assess the effect of competition on small-mammal and desert annual communities. The rodent community on the control

\footnotetext{
${ }^{2}\langle\mathrm{http}: / / \mathrm{www}$.fsl.orst.edu/lter/data/abstract.cfm?dbcode $=$ WE026 $\rangle$

${ }^{3}\langle$ http://culter.colorado.edu/exec/.extracttoolA?smammals.jh $\rangle$

${ }^{4}\langle$ http://sevilleta.unm.edu/data/contents/SEV049/〉
} 


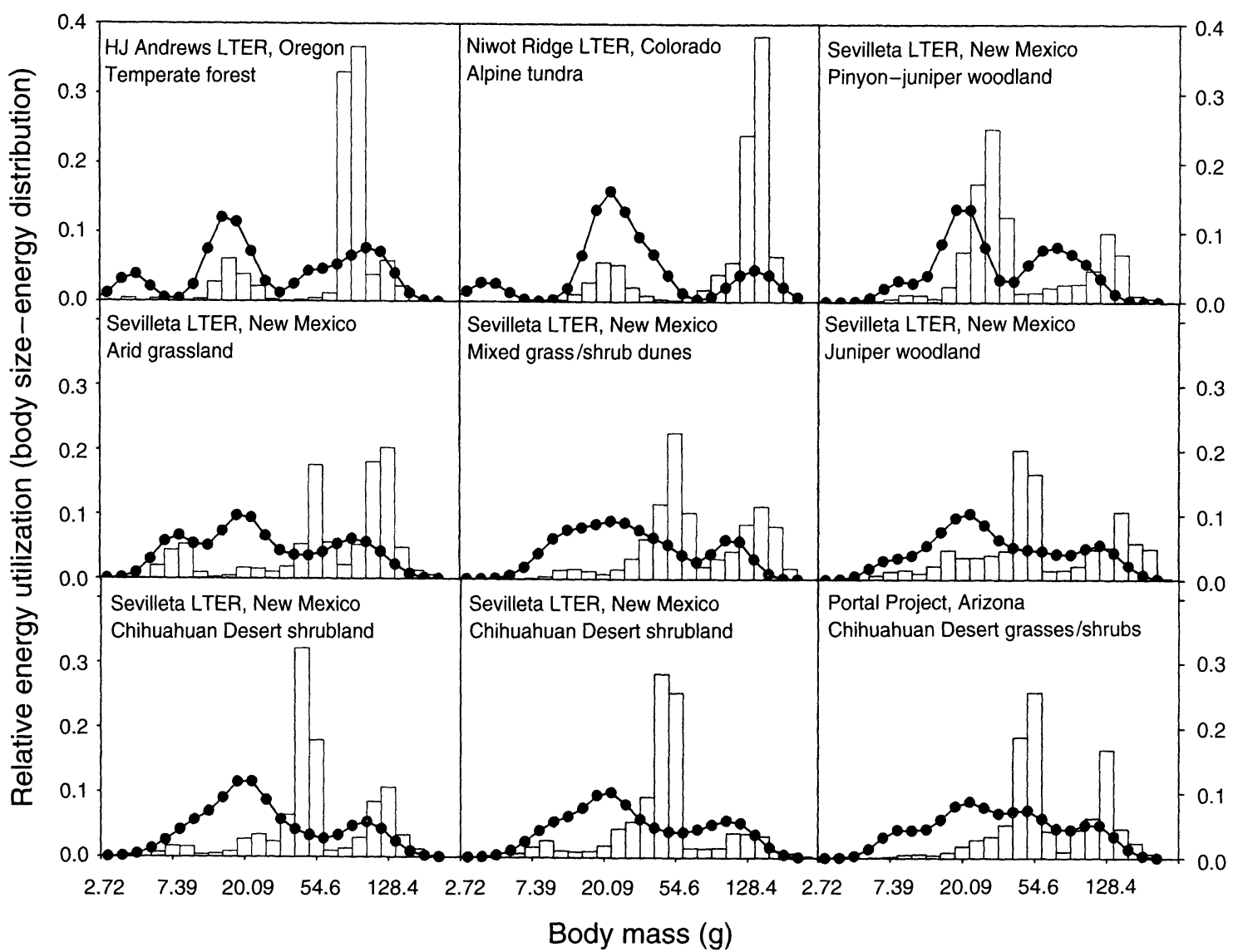

FIG. 1. Body size-energy (white bars) and species-level body size (black circles and line) distributions for nine smallmammal communities. The body size-energy distribution was calculated using an equation for individual metabolic rate based upon body mass. The species-level body size distributions were smoothed using kernel density estimation.

plots from 1977 to 1999 contains 21 species ranging in size from $7.7 \mathrm{~g}$ to $157 \mathrm{~g}$. For a review of the Portal Project, see Brown (1998).

\section{Body size-energy distributions (BSED)}

Energy use in the community can be estimated from individual metabolic rates $(B)$, which can be estimated from body mass $(M)$ using the equation: $\mathrm{B} \propto \mathrm{M}^{3 / 4}$ (Kleiber 1932, Peters 1983, West et al. 1997). $B$ was calculated from this equation for each individual based on its body size. Energetic requirements for individuals were then summed within body size classes (or groupings) of 0.2 natural log units, yielding total energy use of all individuals within a size class, regardless of species identity. This class size was chosen because smaller classes significantly reduce average sample size within classes and larger size classes pool individuals of drastically different body masses. Analyses were also conducted using size classes from 0.1 to $0.3 \mathrm{log}$ units without qualitative changes in the patterns. Energy use through each body size class is reported as a proportion of total community energy use for comparison among communities.

\section{Species-level body size distributions (BSD)}

For statistical tests of BSD, I used the traditional species-based frequency distribution of the mean mass of each species within the community (sensu Hutchinson and MacArthur 1959). I tested the species-level body size distribution against a uniform distribution and compared sites to each other using the Kolmogorov-Smirnov tests. For visual, but not statistical, comparison of the BSD to the BSED, the distribution of mean masses was smoothed using kernel density estimation (Fig. 1; Manly 1996). I used a Gaussian kernel to mimic the actual body size distribution in log space and the average standard deviation of the mean of the logged masses as an estimate for the smoothing parameter, $h$. Sampling points for the kernel density estimation were aligned with the midpoint for each size class in the BSED. This technique was used for comparability to other studies examining aggregations in the species-level body size distribution (i.e., Manly 1996).

\section{Energetic dominance}

Energy use modes were defined as contiguous body size classes in which the energy use of each size class 


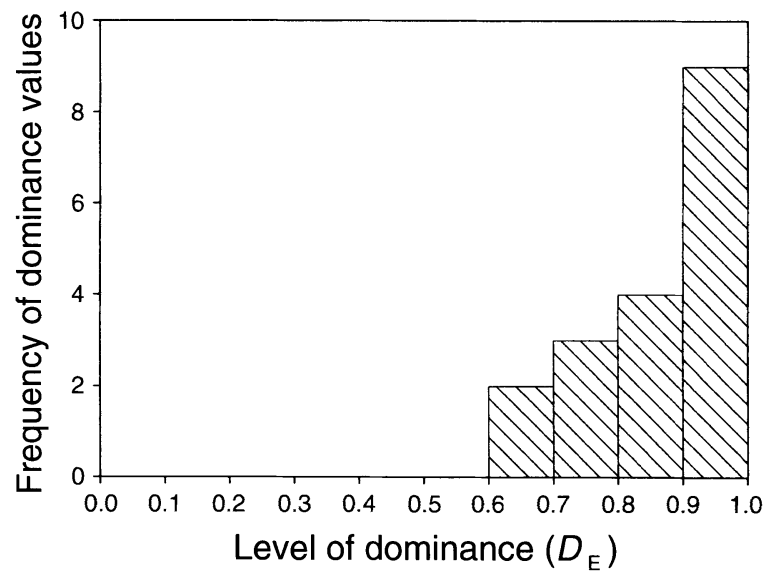

FIG. 2. The frequency distribution of dominance values within energy use modes of the body size-energy distribution for the nine small-mammal communities. The relative dominance of a species within a mode $\left(D_{\mathrm{E}}\right.$, where $\mathrm{E}$ stands for energy) was calculated using the Berger-Parker dominance index (Berger and Parker 1970).

was greater than $5 \%$ of the community total. This percentage is slightly higher than expected if energy were distributed uniformly across body sizes. After identifying energy use modes, the total energy use for each species was calculated. Within an energy use mode, dominance by species with the highest energy use was calculated using a modification of the Berger-Parker dominance index (Berger and Parker 1970), $D_{\mathrm{E}}=p_{\max }$, where $p_{\max }$ is the maximum proportion of energy use by any one species in a mode.

\section{Statistical tests}

Because energy values in the BSEDs represent sums across individuals within a size class and not counts of values for individuals, they are not traditionally defined frequency- or count-based distributions and standard statistical tests of distribution shape are invalid (Sokal and Rohlff 1995). Therefore, BSEDs were tested for uniformity using bootstrap resampling. Random communities were drawn by choosing $n$ uniformly distributed random masses from between the minimum and maximum mass of the community ( $n=$ the total number of individuals within a community). To calculate the similarity or overlap between two body sizeenergy distributions, I used a distribution overlap index (DOI):

$$
\mathrm{DOI}=\sum_{k}\left|\left(y_{a k}-y_{b k}\right)\right|
$$

where $y$ is the value for size class $k$ at study site $a$ or $b$. Because all distributions were normalized, this index ranges from zero (complete overlap) to 2.0 (no overlap). The DOI between each random community and a true uniform distribution was calculated. Ten thousand bootstraps were used to develop a distribution of DOIs expected to occur based on sampling from the uniform. The $P$ value represents the proportion of random-uni- form BSEDs with DOIs greater than the observed distribution. I used a similar technique to compare the BSEDs among communities. Masses of all individuals for the two communities being compared were pooled. From this pool, masses for two resampled communities (one each of the size of the original communities) were drawn randomly and with replacement. A DOI was calculated for the two random communities, and this procedure was repeated 10000 times to create a distribution of expected DOI values for the communities being compared. Significance was determined in the same manner as for uniformity.

BSDs were tested for statistical difference from a uniform distribution using the $\delta$-corrected Kolmogorov-Smirnov goodness of fit test. The $\delta$-corrected Kolmogorov-Smirnov test increases the power of the test when sample sizes are small $(n<25$; Zar 1999). BSD distributions were compared among communities using the Kolmogorov-Smirnov two-sample test (using SPSS). The Kolmogorov-Smirnov test was chosen for these tests because it is sensitive to differences in the overall shape of the distribution (i.e., location, dispersion, skewness; Sokal and Rohlf 1995).

\section{REsults}

BSEDs for all communities were significantly different from uniform (Fig. 1; all $P \leq 0.001$ ). Within energy use peaks, one species often accounted for the majority of energy use, resulting in consistently high values of $D_{\mathrm{E}}$ across all nine communities (range: $0.60-$ 1 , average $D_{\mathrm{E}}=0.85 ;$ Fig. 2). In contrast to the energy distributions, none of the species-level body size distributions were statistically different from uniform $(\delta-$ corrected Kolmogorov-Smirnov goodness of fit test: all $P>0.10$; Appendix A). Visual comparisons of the distributions for each community (Fig. 1) indicate that, while the BSDs at two sites, Niwot and Andrews, appear to reflect their BSEDs (though only in positions of modes, not magnitude) the BSDs in the other seven communities exhibited distinctly different patterns than their respective energy distributions. Together, the uniformity tests of the body size and the body size energy distributions, as well as direct visual inspection, indicate that BSEDs differ from the species-level body size distributions in overall shape.

While sites exhibited the same general multimodal shapes in their BSED, direct comparison of sites revealed that the distributions of all sites were significantly different from one another (all pairwise comparisons of DOI: $P<0.001$ ). In contrast to the BSEDs, all sites had similar species-level body size distributions (Kolmogorov-Smirnov two sample tests: all $P>$ 0.53; for details, see Appendix B). These results are not surprising since all sites also exhibited BSDs that were not significantly different from uniform.

\section{DISCUSSION}

The strong multimodal pattern of energy use within small-mammal communities indicates that energy is 
not utilized equally by different body sizes. The consistency of this pattern across habitats indicates that it may be a common feature of small-mammal communities. Combined with earlier work demonstrating multimodal abundance and biomass distributions (Sheldon et al. 1972, Cyr and Pace 1993, Griffiths 1996), this suggests that multimodal BSEDs are likely to be a general pattern not only within small-mammal communities but across diverse taxa.

However, these results do not support the idea that the structure of a community (i.e., how species, as opposed to individuals, are assembled in a community) reflects the underlying pattern of energy use (Holling 1992). None of the communities exhibited a BSD that was statistically different from uniform (Appendix A, Table A1). However, it should be noted that, because some communities contain fewer species than others, the sample sizes for some communities are small. Examination of the species-level body size distributions (Fig. 1) shows what appear to be aggregations of species along the body size axis. These aggregations were not statistically detectable, but even if they were they would not support the mechanism proposed by Holling (1992) that the aggregations of species on the body size axis should reflect resource availability or use. Seven out of nine communities did not have aggregations of species along the body size axis corresponding to high energy use (Fig. 1). In fact, if the aggregations exist, they tend to correspond to body sizes where energy use is actually low. Niwot and Andrews LTERs are exceptions, with apparent aggregations of species along the body size axis corresponding to high energy use. However, it should also be noted that even for those sites the largest aggregations do not appear to correspond to the largest energy use peaks. In general, the striking differences between the BSD and the BSEDs suggest that the distribution of species along the body size axis within a community does not necessarily correspond to the distribution of mammalian energy use. However, it should be noted that the BSED is a measure of how energy is actually used by the community and not a direct measure of underlying resource availability. Because of this discrepancy between community usage and resource availability, I cannot definitively reject the hypothesis that aggregations of species result from underlying patterns of resource availability.

While the multimodal distribution of energy use is a strong and consistent pattern across communities, it is unclear what the underlying process is. One possible process is that the distribution of energy use follows resource availability. Processes within ecosystems operate on multiple spatial and temporal scales that can result in patchy or fractal-like distributions of resources. This could potentially result in organisms being able to perceive and/or utilize only the subset of resources that are available at the scale at which the organism forages (Holling 1992, Milne 1992, Ritchie 1998). Be- cause many of the characteristics of species that determine the temporal and spatial scales at which an organism operates are closely tied to body size (e.g., generation time, locomotion rates, home range size; Peters 1983), different resources may only be available to organisms of certain sizes. A series of studies conducted on Darwin's finches on the Galapagos have shown that the relationship between abundance and beak size for Darwin's finch is unimodal or multimodal, depending upon the island (Schluter et al. 1985). The shapes of these distributions and the differences between islands appear to result from the underlying distribution of seed sizes (Schluter et al. 1985, Grant 1986, Grant and Grant 1996), suggesting that resource distributions could create the body size energy distributions reported here.

An alternative process to resource availability, which is often assumed to be important in determining community structure, is competition. The possibility that interspecific interactions could affect the body size structure of the community was first postulated by Hutchinson (1959). Coexistence of species in communities is facilitated by resource division (Tilman 1988), and differences in body size in otherwise similar species may facilitate the division of resources and thus promote coexistence (Hutchinson 1959, Bowers and Brown 1982, Grant 1986). For rodents, and desert rodents in particular, body size appears important to structuring communities (Bowers and Brown 1982). In this study, the high degree of dominance exhibited in the energy use modes of the BSEDs also suggests that body size-mediated competition may be important for structuring energy use. An average dominance of $85 \%$ implies that, across an energy use mode, energy is predominately used by a single species. Further, when the species-level body size distribution is compared to the BSED (Fig. 1), aggregations of species do not coincide with the highest energy use, suggesting that there may be strong competition within modes. This high dominance may also have implications for the multi-modal structure of the BSED. If competition is intense between species of similar size, then energetically dominant species cannot coexist within an energy use mode. This process could result in the two or three most energetically dominant species creating distinct body size-energy modes, and thus creating a multimodal BSED through competition. In fact, competition between species of similar size might explain patterns in the BSD reported in this and other studies (e.g., Holling 1992, Allen et al. 1999) that suggest the presence of clusters of species along the body size axis. Finally, it is important to note that neither resource availability nor competition is mutually exclusive. In fact, Schluter and Grant (1984) suggested that while resource distributions can create multimodality, competition should result in exclusive control of resources by one species, resulting in the types of patterns demonstrated here for the rodents. However, while competition and resource 
distributions are frequently assumed to be the important processes driving community structure, they are not the only possible processes which could generate these patterns. Further research is clearly needed to understand what processes are important in creating multimodal body size-energy distributions in small-mammal communities.

While this study focused solely on mammals, it is important to note that mammals are not the only animals involved in energy use in these communities. For example, in North American desert systems (the majority of communities in this study), most rodents are granivores. However, there are two other abundant groups of granivores in these deserts: birds and ants (Brown et al. 1979). Because ants are substantially smaller than $4 \mathrm{~g}$, which is the minimum size of rodents in these communities, we might expect to see additional peaks in energy use.occurring below $4 \mathrm{~g}$ if ants were added to this analysis. The impact of birds is less clear. While there is a high diversity of granivorous birds ir desert ecosystems, seed use in North American deserts tends to be highly dominated by rodents and ants, with birds consuming only a small percentage of available seeds (Brown et al. 1975, Mares and Rosenzweig 1978, Parmenter et al. 1984). This suggests that birds would probably have only a minimal impact on the shape of the BSED for deserts in this body size range. However, because of the lack of comparable density data for birds and rodents in a single community, it is difficult to discern the effects of birds. Field research that intensively censuses multiple taxonomic groups and obtains masses for the majority of individuals is needed not only to assess whether multiple taxonomic groups reinforce the multimodal BSED for a community but also to assess whether different taxonomic groups exhibit a different correspondence between the BSD and the BSED from that shown for these small-mammal communities.

This study indicated strong relationships between energy use, body size, and community structure in smallmammal communities and did not support the idea that energy use is uniform across body size classes. This study also did not support the idea that peaks in energy use result in aggregations of species of similar body sizes within communities. Further exploration of the body size-energy relationship through time and across habitats and taxa should yield additional insights into the processes influencing community structure and dynamics.

\section{ACKNOWLEDGMENTS}

J. Goheen, R. Stevens, N. McIntyre, C. Bloch, J. H Brown, F. Smith, S. Collins, and E. Bedrick provided discussion, comments on the manuscript and/or statistical advice. F. S. Dobson and three anonymous reviewers made substantial and much appreciated contributions to this manuscript. A special thanks to E. P. White for advice, discussion, comments, and patience. Data sets provided by the Sevilleta LTER Data Bank and the Forest Science Data Bank were funded by the NSF LTER program (Sevilleta: BSR- 9411976 and DEB-0080529;
H. J. Andrews: BSR-90-11663 and DEB-96-32921). Data were also provided by the NSF funded Niwot Ridge LTER and the University of Colorado Mountain Research Station. Finally, the Portal Project (J. H. Brown and T. J. Valone, Principle Investigators) is currently supported by NSF LTREB DEB-0348896. S. K. M. Ernest was supported by DEB-0083422.

\section{Literature Cited}

Ackerman, J. L., D. R. Bellwood, and J. H. Brown. 2004. The contribution of small individuals to density-body size relationships: examination of energetic equivalence in reef fishes. Oecologia 139:568-571.

Allen, C., E. Forys, and C. Holling. 1999. Body mass patterns predict invasions and extinctions in transforming landscapes. Ecosystems 2:114-121.

Berger, W. H., and F. L. Parker. 1970. Diversity of planktonic foraminifera in deep-sea sediments. Science 168:13451347.

Blackburn, T. M., V. K. Brown, B. M. Doube, J. J. D. Greenwood, J. H. Lawton, and N. E. Stork. 1993. The relationship between abundance and body size in natural animal assemblages. Journal of Animal Ecology 62:519-528.

Blackburn, T. M., and J. H. Lawton. 1994. Population abundance and body size in animal assemblages. Philosophical Transactions of the Royal Society of London Series B 343: 33-39.

Bowers, M. A., and J. H. Brown. 1982. Body size and coexistence in desert rodents-chance or community structure. Ecology 63:391-400.

Brown, J. H. 1998. The desert granivory experiments at Portal. Pages 71-95 in W. J. Resetarits, Jr. and J. Bernardo, editors. Experimental ecology: issues and perspectives. Oxford University Press, New York, New York, USA.

Brown, J. H., J. J. Grover, D. W. Davidson, and G. A. Lieberman. 1975. A preliminary study of seed predation in desert and montane habitats. Ecology 56:987-992.

Brown, J. H., and P. F. Nicoletto. 1991. Spatial scaling of species composition: body masses of North American land mammals. American Naturalist 138:1478-1512.

Brown, J. H., O. J. Reichman, and D. W. Davidson. 1979. Granivory in desert ecosystems. Annual Review of Ecology and Systematics 10:201-227.

Cyr, H., and M. L. Pace. 1993. Allometric theory-extrapolations from individuals to communities. Ecology 74: 1234-1245.

Damuth, J. 1981. Population-density and body size in mammals. Nature 290:699-700.

Enquist, B. J., and K. J. Niklas. 2001. Invariant scaling relations across tree-dominated communities. Nature 410: 655-660.

Ernest, S. K. M., J. H. Brown, and R. R. Parmenter. 2000. Rodents, plants, and precipitation: spatial and temporal dynamics of consumers and resources. Oikos 88:470-482.

Ernest, S. K. M., et al. 2003. Thermodynamic and metabolic effects on the scaling of production and population energy use. Ecology Letters 6:990-995.

Forys, E. A., and C. R. Allen. 2002. Functional group change within and across scales following invasions and extinctions in the everglades ecosystem. Ecosystems 5:339-347.

Gaston, K. J., and T. M. Blackburn. 2000. Pattern and process in macroecology. Blackwell Science, Oxford, UK.

Grant, P. R. 1986. Ecology and evolution of Darwin's Finches. Princeton University Press, Princeton, New Jersey, USA.

Grant, P. R., and B. R. Grant. 1996. Finch communities in a climatically fluctuating environment. Pages 343-390 in M. L. Cody and J. A. Smallwood, editors. Long-term studies of vertebrate communities. Academic Press, San Diego, California, USA. 
Griffiths, D. 1986. Size-abundance relations in communities. American Naturalist 127:140-166.

Holling, C. S. 1992. Cross-scale morphology, geometry, and dynamics of ecosystems. Ecological Monographs 62:447502.

Horn, H. S., and R. M. May. 1977. Limits to similarity among coexisting competitors. Nature 270:660-661.

Hutchinson, G. E. 1959. Homage to Santa Rosalia; or, why are there so many kinds of animals? American Naturalist 93:145-159.

Hutchinson, G. E., and R. H. MacArthur. 1959. A theoretical ecological model of size distributions among species of animals. American Naturalist 93:117-125.

Istock, C. A. 1973. Population characteristics of a species ensemble of water boatmen. Ecology 54:535-544.

Kerr, S. R., and L. M. Dickie. 2001. The biomass spectrum. Columbia University Press, New York, New York, USA.

Kleiber, M. 1932. Body size and metabolism. Hilgardia 6: 315-353.

Lambert, W. D., and C. S. Holling. 1998. Causes of ecosystem transformation at the end of the Pleistocene: evidence from mammal body-mass distributions. Ecosystems 1:157 175.

Manly, B. F. J. 1996. Are there clumps in body-size distributions? Ecology 77:81-86.

Mares, M., and M. Rosenzweig. 1978. Granivory in North and South American deserts-rodents, birds, and ants. Ecology 59:235-241.

Marquet, P. A., S. A. Navarrete, and J. C. Castilla. 1990. Scaling population-density to body size in rocky intertidal communities. Science 250:1125-1127.

Milne, B. T. 1992. Spatial aggregation and neutral models in fractal landscapes. American Naturalist 139:32-57.

Morse, D. R., N. E. Stork, and J. H. Lawton. 1988. Species number, species abundance and body length relationships of arboreal beetles in Bornean lowland rain forest trees. Ecological Entomology 13:25-37.

Pagel, M. D., P. H. Harvey, and H. C. J. Godfray. 1991 Species-abundance, biomass, and resource-use distributions. American Naturalist 138:836-850.

Parmenter, R. R., J. A. MacMahon, and S. B. Vanderwall. 1984. The measurement of granivory by desert rodents, birds and ants - a comparison of an energetics approach and a seed-dish technique. Journal of Arid Environments 7:75-92.

Peters, R. H. 1983. The ecological implications of body size. Cambridge University Press, Cambridge, UK.
Ritchie, M. E. 1998. Scale-dependent foraging and patch choice in fractal environments. Evolutionary Ecology 12: 309-330.

Russo, S., S. Robinson, and J. Terborgh. 2003. Size-abundance relationships in an Amazonian bird community: implications for the energetic equivalence rule. American Naturalist 161:267--283.

Schluter, D., and P. R. Grant. 1984. Determinants of morphological patterns in communities of Darwin's Finches. American Naturalist 123:175-196.

Schluter, D., T. D. Price, and P. R. Grant. 1985. Ecological character displacement in Darwin's Finches. Science 227: 1056-1059.

Sheldon, R. W., A. Prakash, and W. H. J. Sutcliffe. 1972. The size distribution of particles in the ocean. Limnology and Oceanography 17:327-340.

Siemann, E., and J. H. Brown. 1999. Gaps in mammalian body size distributions reexamined. Ecology 80:27882792.

Silva, M., and J. A. Downing. 1995. The allometric scaling of density and body mass: a nonlinear relationship for terrestrial mammals. American Naturalist 145:704-727.

Sokal, R. R., and F. J. Rohlf. 1995. Biometry. Third edition. W. H. Freeman and Company, New York, New York, USA.

Stork, N. E., and T. M. Blackburn. 1993. Abundance, body size and biomass of arthropods in tropical forest. Oikos 67: 483-489.

Strong, D. R., L. A. Szyska, and D. Simberloff. 1979. Tests of community-wide character displacement against null hypotheses. Evolution 33:897-913.

Taper, M., and P. Marquet. 1996. How do species really divide resources? American Naturalist 147:1072-1086.

Thibault, K. M., E. P. White, and S. K. M. Ernest. 2004. Temporal dynamics in the structure and composition of a desert rodent community. Ecology 85:2649-2655.

Tilman, D. 1988. Plant strategies and the dynamics and structure of plant communities. Princeton University Press, Princeton, New Jersey, USA.

West, G. B., J. H. Brown, and B. J. Enquist. 1997. A general model for the origin of allometric scaling laws in biology. Science 276:372-373.

White, E. P., S. K. M. Ernest, and K. M. Thibault. 2004. Tradeoffs in community properties through time in a desert rodent community. American Naturalist 164:670-676.

Zar, J. H. 1999. Biostatistical analysis. Fourth edition. Prentice Hall, Englewood Cliffs, New Jersey, USA.

\section{APPENDIX A}

A table of test statistics for $\delta$-corrected Kolmogorov-Smirnov tests for comparisons of the species-level body size distributions to a uniform distribution is available in ESA's Electronic Data Archive: Ecological Archives EO86-076-A1.

\section{APPENDIX B}

A table of test statistics for Kolmogorov-Smirnov two-sample tests comparing the species-level body size distributions between sites is available in ESA's Electronic Data Archive: Ecological Archives EO86-076-A2. 